\title{
Sélection thématique de livres
}

Cette rubrique signale les ouvrages récemment parus en reprenant la quatrième de couverture ou la présentation des éditeurs. Les livres sont classés en trois catégories: les ouvrages thématiques par objet d'étude; les ouvrages sur la science et ses rapports avec la société; les ouvrages de réflexion sur les sciences.

\section{Agriculture, alimentation}

Des « mai 68 » dans les campagnes françaises? Les contestations paysannes dans les années 1968 Jean-Philippe Martin

L'Harmattan, 2017, $236 \mathrm{p}$.

Les années 1968 évoquent les étudiants, quelquefois les ouvriers mais rarement les agriculteurs. Pourtant, des luttes paysannes majeures, souvent oubliées, se déroulent dans l'Ouest et le Languedoc-Roussillon. Elles sont massives, fréquentes et novatrices : grève du lait, " guerre du vin », lutte du Larzac... Certaines prennent même une dynamique régionaliste et trouvent un large écho dans la société.

Des paysans contestataires remettent en cause les syndicats majoritaires, défendent la nécessité d'un syndicalisme d'action démocratique, refusent le corporatisme, entrent en lutte contre la politique agricole des gouvernements, voire se disent anticapitalistes. D'autres, moins engagés, empruntent au registre de la contestation et en reprennent le répertoire d'actions, les thèmes ou les alliances.

Pourquoi et comment émerge un courant contestataire dans le monde paysan? Qu'est-ce qui fait sa spécificité? Quelles ont été ses évolutions? Que reste-t-il aujourd'hui des débats portés par ces contestations? En quoi la Confédération paysanne en est-elle l'héritière ? Voilà les principales questions abordées par cet ouvrage.

\section{Les grands fermiers. $\mathrm{XV}^{\mathrm{e}}-\mathrm{XVIII}{ }^{\mathrm{e}}$ siècle}

Jean-Marc Moriceau

Fayard, 2017, 512 p.

Modèle emblématique des grands exploitants, les fermiers de l'île-de-France ont façonné les paysages de nos plaines, modelé l'agriculture, décrit d'étonnantes trajectoires sociales. Leurs descendants sont innombrables.

Cette fresque de plus de trois siècles a contribué à la relance de l'histoire rurale et à l'essor d'une histoire sociale soucieuse du changement dans la longue durée. Pour l'historien, le sociologue, le démographe et le géographe, pour l'économiste, le littéraire ou l'anthropologue, pour l'observateur du monde agricole et le public curieux du passé rural ou simplement en quête de racines, ce texte qui fait référence est désormais accessible.

\section{L'histoire de l'Inra, entre science et politique}

Pierre Cornu, Egizio Valceschini, Odile Maeght-Bournay Quæ, 2017, 464 p.

Alors que le monde industrialisé pensait en avoir fini avec la question des subsistances, la Seconde Guerre mondiale replace l'agriculture et l'alimentation au cœur des enjeux de la reconstruction. L'Europe de l'Ouest, ravagée et affaiblie, se tourne alors vers les États-Unis pour penser une modernisation capable de fonder une nouvelle expansion. En France, l'après-guerre est ainsi le théâtre d'une profonde transformation du monde rural sous l'effet de la diffusion volontariste du "progrès », instrument d'une mise à niveau de la productivité agricole au service de la restauration de la position française dans le monde. Dans ce contexte, la création en 1946 de l'Inra constitue un moment crucial de la rencontre entre science et politique, dans la quête d'une voie de développement qui mobilise les outils des sciences du vivant pour en rationaliser l'exploitation.

D'abord conçu comme un institut de recherche et d'expérimentation dévolu à la seule agriculture, l'Inra élargit peu à peu ses missions, déployant des compétences nouvelles sur les industries agroalimentaires, la nutrition, la biologie moléculaire ou encore l'environnement. Serviteur zélé des politiques publiques de modernisation, l'institut s'affirme dans le concert des grands opérateurs de recherche, aux côtés du CNRS, de l'Inserm et de l'Institut Pasteur. Mais confronté à l'essor de la recherche privée, à l'effacement du volontarisme d'État et à la montée de la contestation des sciences et des techniques, il se trouve précocement contraint, dans les 
années 1980 et surtout 1990, à repenser en profondeur son identité et son rôle, dans la prise de conscience croissante de l'interconnexion des questions agricoles, alimentaires et environnementales.

Au cœur des enjeux de l'économie de la connaissance du monde contemporain, la trajectoire historique de l'Inra représente une véritable énigme, observatoire privilégié de la genèse d'une gouvernance globale du vivant et de la sécurité alimentaire. Fondé sur une documentation originale et sur un recours à la mémoire des acteurs, le présent ouvrage constitue la première synthèse historique sur cet organisme de recherche.

\section{Nourrir l'Europe en temps de crise. Vers des systèmes alimentaires résilients Pablo Servigne}

Actes Sud, 2017, 208 p.

En se basant sur les études scientifiques les plus pointues, Pablo Servigne propose une synthèse sans précédent et sans concession de la toxicité et surtout de la vulnérabilité de notre système alimentaire industriel. De la production à la transformation, de la distribution à la gestion des déchets, celui-ci est entièrement dépendant du pétrole et donc inévitablement voué à disparaître avec la fin des énergies fossiles.

À quoi pourraient alors ressembler les systèmes alimentaires de demain? En présentant quelques alternatives qui ont prouvé qu'elles n'étaient ni farfelues ni anecdotiques - de la permaculture à l'agriculture urbaine en passant par l'agroécologie ou le retour à la traction animale-, Pablo Servigne démontre, avec rigueur et conviction, que nous sommes à l'aube d'un changement radical et inévitable dans nos manières de faire et de penser.

\section{Le nouveau capitalisme agricole.}

De la ferme à la firme

François Purseigle, Geneviève Nguyen, Pierre Blanc (Eds) Les Presses de Sciences Po, 2017, 306 p.

En rupture avec l'exploitation familiale traditionnelle, des firmes agricoles se profilent dans de nombreuses régions du globe. Avec elles s'amorce une recomposition agraire dont les contours restent largement invisibles. À l'exception de quelques cas médiatisés de «mégafermes » et autres «fermes des 1000 vaches», la majorité de ces firmes ont jusqu'à présent échappé à l'analyse tant leurs visages sont multiples et flous.

Leurs performances économiques et financières tout comme leurs incidences environnementales et sociales interrogent aussi bien les milieux politiques, professionnels que scientifiques. Comment sont-elles organisées? Quels sont leurs logiques et modes d'action? Dans quels espaces s'inscrivent-elles? Comment une ferme familiale devient-elle une firme agricole? Quels types d'acteurs sont impliqués? Quels rapports au politique entretiennent-elles? Quels conflits provoquent-elles?

Le premier ouvrage sur un phénomène mondial en pleine émergence.

\section{Changement climatique}

\section{Le Capitalocène.}

Aux racines historiques du dérèglement climatique Armel Campagne

Éditions Divergences, 2017, 210 p.

Le dérèglement climatique nous aurait fait entrer dans "l'Anthropocène», cette ère géologique durant laquelle l'espèce humaine serait devenue la force écologique majeure. Mais le dérèglement climatique est-il réellement imputable à l'«espèce humaine» de manière indifférenciée et prise comme espèce naturelle? Des historiens ont mis en doute cette vision simpliste, en faisant de l'industrialisation - et donc du dérèglement climatique - un processus résultant avant tout des dynamiques du capitalisme, inventant à cette occasion un nouveau concept: le Capitalocène. Cet ouvrage effectue un panorama critique des propositions de ces historiens en vue d'une nouvelle histoire des causes historiques du dérèglement climatique.

\section{Global climate justice. \\ Proposals, arguments and justification}

Olivier Godard

Edward Elgar, 2017, 232 p.

In this thoughtful and original book, social scientist Olivier Godard considers the ways in which arguments of justice cling to international efforts to address global climate change. Proposals made by governments, experts and NGOs as well as concepts and arguments born of moral and political philosophy are introduced and critically examined. Godard contributes to this important debate by showing why global climate justice is still controversial, despite it being a key issue of our times.

Godard first points out the huge differences between the foundations of conflicting proposals, for instance between a cosmopolitan viewpoint and an international one. He then explores controversies over climate justice proposals and provides a rigorous criticism of those based on historical responsibility. Finally, he demonstrates how issues of justice are reconfigured by instrumental regimes of coordination, such as a global carbon market. Inspired by the French school of justification, this book shines an insightful light on the failure of climate change debates to develop a convincing standard moral and political theory.

Including elements from systems theory, economics and law, this book will be of interest to scholars and 
students of moral and political philosophy, economics and social sciences, as well as experts working on climate negotiations and concerned stakeholders.

Heat, greed and human need.

Climate change, capitalism and sustainable wellbeing Ian Gough

Edward Elgar, 2017, 264 p.

This exceptional book considers how far catastrophic global warming can be averted in an economic system that is greedy for growth, without worsening deprivation and inequality. The satisfaction of human needs - as opposed to wants - is the only viable measure for negotiating trade-offs between climate change, capitalism and human wellbeing, now and in the future.

The author critically examines the political economy of capitalism and offers a long-term, interdisciplinary analysis of the prospects for keeping the rise in global temperatures below two degrees, while also improving equity and social justice. A three-stage transition is proposed with useful practical policies. First, 'green growth': cut carbon emissions from production across the world. Second, 'recompose' patterns of consumption in the rich world, cutting high-energy luxuries in favour of low-energy routes to meeting basic needs. Third, because the first two are perilously insufficient, move towards an economy that flourishes without growth.

Heat, greed and human need is vital for researchers and students of the environment, public and social policy, economics, political theory and development studies. For those advocating political, social and environmental reform this book presents excellent practical eco-social policies to achieve both sustainable consumption and social justice.

\section{Pour éviter le chaos climatique et financier}

Jean Jouzel, Pierre Larrouturou

Odile Jacob, 2017, 432 p.

Et si préserver notre climat était l'un des meilleurs moyens d'endiguer la prochaine crise financière? Pour sauver les banques, on a mis 1000 milliards. Pourquoi ne pas mettre 1000 milliards pour sauver le climat? Avec ce livre, le climatologue Jean Jouzel et l'économiste Pierre Larrouturou proposent un vrai Pacte finance-climat européen pour diviser par 4 les émissions de $\mathrm{CO} 2$, dégonfler la bulle financière et créer plus de 5 millions d'emplois.

La machine climatique est en train de s'emballer dangereusement. Il ne nous reste que 3 ans pour inverser la courbe des émissions de gaz à effet de serre si nous voulons éviter aux jeunes d'aujourd'hui un climat auquel il leur serait difficile, voire impossible, de s'adapter. Or, dans le même temps, l'endettement mondial atteint un niveau inédit, les banques centrales nourrissent la spéculation et tout annonce une crise pire que celle de 2008. Favoriser la spéculation ou sauver le climat? À nous de choisir.

\section{Développement durable et transitions}

\section{Ce que tout écologiste doit savoir à propos du capitalisme}

Fred Magdoff, John Bellamy Foster

Éditions Critiques, 2017, 200 p.

Si la crise écologique majeure que nous connaissons est la conséquence des activités humaines, alors une réaction efficace à cette crise implique nécessairement une remise en cause du mode d'activité propre à l'humanité contemporaine, à savoir le capitalisme. Au fil d'un exposé accessible au plus grand nombre, mobilisant avec clarté les données scientifiques et économiques, les auteurs éclairent les enjeux proprement politiques unissant question économique et question écologique.

Ils établissent ainsi l'impossible coexistence d'un mode de production fondé sur l'accumulation et un environnement humainement viable. La dégradation de l'environnement n'est pas un accident, elle s'inscrit au contraire dans la logique du capitalisme. De fait, la promesse d'un « capitalisme vert», ou l'espoir d'un salut écologique par les nouvelles technologies conservant l'ordre économique actuel, ne peuvent être qu'illusions.

Écologie intégrale. Pour une société permacirculaire Christian Arnsperger, Dominique Bourg

Presses universitaires de France, 2017, 204 p.

Il faut bien le constater, la transition numérique mondiale s'accélère tandis que la transition écologique, au mieux, marque le pas. Cette situation ne sera pas longtemps soutenable. Il importe de se donner le plus rapidement possible un objectif collectif qui corresponde enfin à ce qu'exige l'état de dégradation du système Terre. Comment concilier une empreinte écologique radicalement réduite avec la pluralité actuelle de nos sociétés et de leur tissu économique?

Chercher une voie de sortie, c'est changer de regard: en partant d'où nous sommes, sans nier nos désaccords sur les solutions à apporter, envisageons une pluralité de voies d'expérimentation - des micro-expériences citoyennes de permaculture jusqu'à la production industrielle la plus high-tech en passant par les chemins de l'économie sociale et solidaire. À chacune de démontrer que ses solutions permettront d'atteindre l'objectif ultime : une empreinte écologique décroissante pour nous permettre de retourner puis de rester à l'intérieur des limites de la biosphère, sans renoncer à notre modernité, en œuvrant en faveur d'une priorité environnementale enfin claire et, à terme, libératrice. 
Insoutenables inégalités.

\section{Pour une justice sociale et environnementale} Lucas Chancel

Les Petits matins, 2017, $184 \mathrm{p}$.

Dans un contexte d'accroissement des inégalités et de chômage de masse, les politiques environnementales sont souvent perçues comme des contraintes supplémentaires, quand elles ne sont pas qualifiées de mesures anti-pauvres ou anti-ruralité. Pourtant, il existe un lien étroit entre les injustices sociales et environnementales.

En effet, les données chiffrées sont sans appel: au Nord comme au Sud, les plus riches sont les principaux pollueurs, tandis que les plus modestes sont davantage exposés aux risques et plus vulnérables face aux dégâts occasionnés, comme les récents ouragans en Atlantique l'ont montré.

C'est pourquoi la question de la justice sociale doit être mise au cœur des politiques de développement durable. Infrastructures, systèmes de mesure innovants, réformes fiscales... Les solutions et les exemples à suivre ne manquent pas. Seulement, leur mise en œuvre ne se fait pas du jour au lendemain, ni sans résistances, que ce soit en Europe, aux États-Unis ou en Inde. Afin d'accompagner et d'accélérer la nécessaire métamorphose de l'État social, l'auteur propose plusieurs pistes concrètes et plaide pour une meilleure articulation des luttes locales et de la coordination internationale.

\section{Transitions économiques.}

En finir avec les alternatives dérisoires

Philippe de Leener, Marc Totté

Éditions du Croquant, 2017, 312 p.

N'avons-nous pas le sentiment que nous n'en finissons pas de reproduire l'économie que nous croyons combattre? Que notre créativité, nos audaces, nos sacrifices ne cessent paradoxalement de redonner vigueur et jeunesse à cette économie qui nous domine? Qu'elle nous enferme dans des luttes sans fin et des inégalités de plus en plus blessantes? Comment éviter que sa domination en vienne à se perpétuer aussi grâce à «nos alternatives»? Quelle transition engager vers une tout autre économie et comment la mener?

Les auteurs décortiquent d'abord les logiques et pratiques qui nous épuisent dans le «non-changement». Ensuite ils prennent position: seules les luttes concrètes qui s'attaquent délibérément aux fondements de l'économie dominante échappent à la dérision et ouvrent véritablement une nouvelle ère. Ce faisant, ils explorent la perspective d'une économie qui vise l'utilité pour la société et généralise la «prévenance». Ils remettent ainsi l'économie au cœur du champ politique.

L'ouvrage ne propose ni recette, ni modèle, ni prophétie et s'émancipe du clivage gauche-droite. Il met à la portée de tous des questions concrètes, directes et claires qui font le pari de l'intelligence collective.

\section{Un défi pour la planète.}

Les Objectifs de développement durable en débat

Patrick Caron, Jean-Marc Châtaigner (Eds)

Quæ, 2017, 466 p.

" Transformer les vies tout en préservant la planète», telle est l'ambition des 17 Objectifs de développement durable (ODD), cadre d'action à l'échelle planétaire adopté par tous les pays membres de l'Onu en septembre 2015. Universels, les ODD s'adressent autant aux pays du Nord qu'aux pays du Sud et concernent à la fois l'économie, le développement des sociétés et la protection de l'environnement. Lutte contre la pauvreté, égalité entre les sexes, accès de tous à une énergie propre et à une éducation de qualité, bonne santé et bien-être, villes durables, consommation et production responsables... c'est à un profond changement de modèle que nous invitent les ODD. Cette transformation passe par un dialogue renouvelé entre science, société et politique et entraîne des mutations majeures dans les rapports Nord-Sud.

Proposant une analyse critique de chacun des 17 Objectifs, de leurs interactions et des contradictions dont ils sont aussi porteurs, cet ouvrage réunit une soixantaine d'auteurs d'horizons divers: responsables d'institutions scientifiques, chercheurs, experts, acteurs $\mathrm{du}$ développement... Cette approche plurielle offre différentes clés de lecture et met en avant l'importance de la recherche pour comprendre les enjeux des ODD, mesurer l'avancement de leur mise en œuvre et concevoir, en lien avec la société, des solutions innovantes. Accessible à un large public, il apporte un éclairage inédit, à la fois critique et documenté, sur les grands défis de l'Agenda 2030 pour le développement durable.

\section{Écologie, biodiversité, évolution}

\section{L'océan à découvert}

Agathe Euzen, Françoise Gaill, Denis Lacroix, Philippe Cury (Eds)

CNRS Éditions, 2017, 322 p.

Il couvre $70 \%$ de la surface du globe, régule en grande partie le climat de la Terre et abrite une multitude d'espèces dont de très nombreuses sont encore à découvrir. L'océan joue un rôle majeur dans le fonctionnement de la vie sur Terre. Avec le changement global ont émergé de multiples enjeux dont les conséquences à venir engagent toute l'humanité.

Il s'agit dans ce livre de mieux comprendre ce qu'est l'océan, dans toute son étendue, sa profondeur et sa complexité, tant du point de vue physique que biologique, de ses interactions que de ses dynamiques. Pour l'explorer, 
de nombreux outils, infrastructures et approches originales sont développés pour obtenir les données et les informations indispensables à une meilleure connaissance. L'histoire montre l'évolution des techniques et des représentations qui lient les communautés humaines aux océans et la diversité des usages qu'elles en font. Considéré comme ressource, service ou enjeu de territoire, l'océan est essentiel, mais vulnérable. De multiples risques lui sont associés, de la submersion à la pollution, et leur gestion nécessite d'être anticipée face à l'augmentation des activités humaines. Ainsi se pose la question de l'avenir de l'océan, cet espace considéré souvent comme infini et illimité, qualifié de géostratégie par les uns et de bien commun par d'autres.

Avec ses 135 articles, L'océan à dévouvert, qui mobilise près de 160 chercheurs et spécialistes, est un ouvrage de référence. Il vient éclairer des enjeux actuels en résonnance avec les objectifs du développement durable de l'Onu. Comprendre et proposer des solutions, telle est son intention.

\section{Les politiques de biodiversité}

Daniel Compagnon, Estienne Rodary (Eds)

Presses de sciences Po, 2017, 256 p.

La biodiversité est omniprésente dans les arènes nationales et internationales. Affranchie d'une approche exclusivement écologique, elle est devenue un enjeu d'action publique. C'est à cette mise en politique de la biodiversité que s'intéresse cet ouvrage pluridisciplinaire, en reliant des champs scientifiques éclatés et en traitant d'aspects négligés tels que les effets d'échelle, l'impact politique des catégories scientifiques mobilisées par les acteurs et l'instrumentalisation par les États des normes internationales. Ce panorama inédit laisse entrevoir, malgré l'urgence affirmée par les scientifiques et l'enjeu social incontournable qu'elles représentent, un avenir incertain pour les politiques de biodiversité.

\section{Gestion des ressources}

\section{Perspectives on commoning.}

Autonomist principles and practices

Guido Ruivenkamp, Andy Hilton (Eds)

The University of Chicago Press, 2017, 352 p.

In the wake of socialism's demise and liberalism's loss of direction, new ideas are needed for the next major realignment of the social and political domain. Making a unique contribution to the idea of the commons, this book offers a radical new model for direct democracy. In contrast to current scholarship that has looked at the commons from the perspective of governance, this book instead focuses on the idea of commoning as social practice. Perspectives on commoning argues that the commons are not just resources external to us, but are a function of what we do.
Covering everything from biopolitics to communication technologies, urban spaces to agricultural sovereignty, the contributors to this volume address the commons as both theory and history, providing a useful review of current conceptions as well as practical proposals for the future. A unique consolidation of political philosophy, sociology and economics, the book shows how a new understanding of the commons as practice will help to achieve its full emancipatory potential.

\section{Gestion et politiques de l'environnement}

\section{Climate of capitulation.}

An insider's account of state power in a coal nation Vivian E. Thomson

The MIT Press, 2017, 256 p.

The United States has pledged to the world community a reduction in greenhouse gas emissions by $26-28$ percent below 2005 levels in 2025. Because much of this reduction must come from electric utilities, especially coal-fired power plants, coal states will make or break the U.S. commitment to emissions reduction. In Climate of capitulation, Vivian Thomson offers an insider's account of how power is wielded in environmental policy making at the state level. Thomson, a former member of Virginia's State Air Pollution Control Board, identifies a "climate of capitulation" in state government - a deeply rooted favoritism toward coal and electric utilities in states' air pollution policies.

Thomson narrates three cases involving coal and air pollution from her time on the Air Board. She illuminates the overt and covert power struggles surrounding air pollution limits for a coal-fired power plant just across the Potomac from Washington, for a controversial new coal-fired electrical generation plant in coal country, and for coal dust pollution from truck traffic in a country hollow. Thomson links Virginia's climate of capitulation with campaign donations that make legislators politically indebted to coal and electric utility interests, a traditionalistic political culture tending to inertia, and a part-time legislature that depended on outside groups for information and bill drafting. Extending her analysis to fifteen other coal-dependent states, Thomson offers policy reforms aimed at mitigating the ingrained biases toward coal and electric utilities in states' air pollution policy making.

Démanteler les barrages pour restaurer les cours d'eau. Controverses et représentations

Régis Barraud, Marie-Anne Germaine (Eds)

Quæ, 2017, 260 p.

Avec l'application de la directive-cadre européenne sur l'eau, les opérations de restauration écologique des cours d'eau se multiplient depuis les années 2000. 
Cet ouvrage aborde les actions les plus répandues en France, mais aussi les plus controversées, qui consistent à restaurer la continuité écologique des cours d'eau en démantelant des barrages et des seuils, considérés comme des obstacles au transit sédimentaire et à la circulation des poissons migrateurs.

Les débats et conflits engendrés par ces opérations sont analysés dans le contexte spécifique des petites rivières du nord-ouest de la France, qui se distinguent par une forte densité d'ouvrages hydrauliques. À partir d'enquêtes et d'études de cas détaillées, les auteurs, géographes, reconstituent l'histoire de ces aménagements devenus encombrants. Ils contribuent également à mieux saisir les attentes des divers acteurs (élus, gestionnaires, pêcheurs, propriétaires de moulins, riverains, usagers) vis-à-vis de la rivière et rendent compte des décalages entre leurs représentations des paysages fluviaux. Enfin, les auteurs ébauchent une réflexion sur les modalités de construction d'un nouveau projet écologique pour les vallées.

Ce livre s'adresse aux gestionnaires de milieux aquatiques et aux acteurs de la protection des milieux et des ressources en eau, mais aussi aux étudiants en géographie, en écologie, ainsi qu'à des chercheurs intéressés par les recherches interdisciplinaires sur les politiques publiques environnementales et leurs interactions avec les ONG et les associations d'usagers.

\section{Hommes et milieux}

\section{Le champignon de la fin du monde. Sur les possibilités} de vivre dans les ruines du capitalisme

Anna Lowenhaupt Tsing

Les empêcheurs de penser en rond/La Découverte, 2017, $416 \mathrm{p}$.

Ce n'est pas seulement dans les pays ravagés par la guerre qu'il faut apprendre à vivre dans les ruines. Car les ruines se rapprochent et nous enserrent de toute part, des sites industriels aux paysages naturels dévastés. Mais l'erreur serait de croire que l'on se contente d'y survivre. Dans les ruines prolifèrent en effet de nouveaux mondes qu'Anna Tsing a choisi d'explorer en suivant l'odyssée étonnante d'un mystérieux champignon qui ne pousse que dans les forêts détruites.

Suivre les matsutakes, c'est s'intéresser aux cueilleurs de l'Oregon, ces travailleurs précaires, vétérans des guerres américaines, immigrés sans papiers, qui vendent chaque soir les champignons ramassés le jour et qui termineront comme des produits de luxe sur les étals des épiceries fines japonaises. Chemin faisant, on comprend pourquoi la «précarité » n'est pas seulement un terme décrivant la condition des cueilleurs sans emploi stable mais un concept pour penser le monde qui nous est imposé. Suivre les matsutakes, c'est apporter un éclairage nouveau sur la manière dont le capitalisme s'est inventé comme mode d'exploitation et dont il ravage aujourd'hui la planète.

Suivre les matsutakes, c'est aussi une nouvelle manière de faire de la biologie: les champignons sont une espèce très particulière qui bouscule les fondements des sciences du vivant. Les matsutakes ne sont donc pas un prétexte ou une métaphore, ils sont le support surprenant d'une leçon d'optimisme dans un monde désespérant.

\section{Ville}

La ville végétale. Une histoire de la nature en milieu urbain (France, $\mathrm{XVII}^{\mathrm{e}}$-XXI ${ }^{\mathrm{e}}$ siècle)

Charles-François Mathis, Émilie-Anne Pépy

Champ Vallon, 2017, $360 \mathrm{p}$.

Cette vaste synthèse étudie l'occupation urbaine depuis le $\mathrm{XVII}^{\mathrm{e}}$ siècle par les plantes, parcs et jardins, mais aussi les plantes «hors-sol» qui circulent dans l'espace urbain (fruits et légumes, fleurs coupées, déchets végétaux, etc.). Il s'agit de montrer que loin d'être un espace absolument artificialisé, non «naturel», la ville a constamment été imprégnée de végétaux, mais que cette présence a fluctué au fil des siècles. Sont interrogés les raisons, les acteurs et les défis de la végétalisation urbaine qui repose sur des motivations nombreuses, esthétiques, sanitaires, écologiques. De même, la végétalisation a sans cesse été soutenue par ceux que nous appelons les mains vertes, qui sont tout aussi bien des particuliers, des professionnels que les pouvoirs publics. Mais étendre ou maintenir la présence végétale en ville impose de relever de multiples défis (fonciers, financiers, esthétiques, biologiques...) qui ont pu varier au cours des siècles.

Ce livre propose aussi une réflexion neuve sur les usages qui ont été faits de la nature en ville, paradoxalement instrument d'urbanité, de sociabilité et donc d'affirmation sociale, mais aussi inversement jungle, ferment de discorde et d'ensauvagement, objet d'affrontements politiques. On peut également faire usage du végétal pour bien être et bien vivre, accompagner les plaisirs, exercer les corps ou cultiver les esprits. Plus prosaïquement, la ville est aussi, tout au long de ces quatre siècles, un lieu de production et de consommation important de végétal. Enfin, c'est aussi en ville qu'une science botaniste et un savoir sur les plantes se développent. Ce tableau général, inédit, permet ainsi de voir la ville autrement et d'offrir des pistes de réflexion sur la nature en milieu urbain aujourd'hui. 


\section{Histoire et philosophie des sciences}

\section{Pour une philosophie de l'Anthropocène}

Alexander Federau

Presses universitaires de France, 2017, 436 p.

La pression que les activités humaines font peser sur le système Terre s'accroît si vite que les sciences de la Terre annoncent l'entrée dans une nouvelle époque géologique. L'Anthropocène, Âge de l'homme, est ainsi le temps où les effets conjugués de la consommation, de la technologie et de la démographie deviennent la force géologique dominante.

Mais au croisement des temps géologique et historique, le récit que propose l'Anthropocène est controversé. Sommes-nous les héros de cette nouvelle époque? Ou les bâtisseurs d'un nouvel Âge de pierre, avec une planète de plus en plus hostile et un accès raréfié aux ressources? Finalement, qui est cet homme que mettent en scène les discours sur l'Anthropocène?

En montrant que nous arrivons au terme d'un long parcours, celui de la modernité, cet ouvrage ouvre une réflexion philosophique sur un monde où l'entrelacs nature-société est devenu inextricable.

Technicité versus scientificité. Tensions et équivoques Giulia Anichini, Flavia Carraro, Philippe Geslin, Georges Guille-Escuret

ISTE éditions, 2017, 206 p.

La relation entre techniques et sciences est étrangement négligée, évitée ou confiée à des intuitions discrètes, bien que sa clarification soit déterminante, notamment pour toute programmation d'une interdisciplinarité. Technicité versus scientificité montre que cette relation, sitôt qu'on l'interroge au lieu de la contourner, se révèle omniprésente dans les sciences, extrêmement diversifiée parmi elles et très modulable au cours des phases d'une recherche.

Quatre anthropologues proposent ici des approches très différentes: ils ne prétendent pas épuiser le sujet, mais au moins montrer que ce contournement régulier est lourd de conséquences et qu'un vaste chantier devrait renaître à cet endroit.

\section{Rapports sciences, technologies et sociétés}

Sciences et société : répondre ensemble aux enjeux climatiques

Julien Blanchet, Jean-Jouzel

Les éditions des Journaux officiels, 2017, 97 p.

Phénomène étudié depuis les années 1970, le réchauffement climatique nécessite un effort soutenu de recherche en vue de mieux comprendre les mécanismes à l'œuvre et d'identifier des solutions pour atténuer son développement et ses impacts. Cette recherche, longtemps œuvre unique des chercheurs, s'ouvre depuis plusieurs années aux acteurs de la société civile (acteurs socioéconomiques, environnementaux, citoyens, décideurs politiques) qui souhaitent y participer aux côtés de chercheurs demandeurs de plus d'interactions avec la société civile. Ce dialogue devrait contribuer à une recherche plus performante sur de nombreux aspects, estime le Conseil économique, social et environnemental (CESE). Dans cet avis, le CESE formule un ensemble de préconisations visant à le renforcer et à le systématiser à travers la création de lieux, l'amélioration de sa gouvernance et sa prise en compte dans le financement de la recherche. Il recommande également l'inclusion au plus haut niveau de l'État d'un éclairage scientifique dans le processus de décision.

\section{Sciences de la vie et de la nature}

Jamais seul. Ces microbes qui construisent les plantes, les animaux et les civilisations

Marc-André Selosse

Actes Sud, 2017, 368 p.

Nous savons aujourd'hui que les microbes ne doivent plus seulement être associés aux maladies ou à la décomposition. Au contraire, ils jouent un rôle en tout point essentiel: tous les organismes vivants, végétaux ou animaux, dépendent intimement de microbes qui contribuent à leur nutrition, leur développement, leur immunité ou même leur comportement. Toujours pris dans un réseau d'interactions microbiennes, ces organismes ne sont donc... jamais seuls.

$\mathrm{Au}$ fil d'un récit foisonnant d'exemples et plein d'esprit, Marc-André Selosse nous conte cette véritable révolution scientifique. Détaillant d'abord de nombreuses symbioses qui associent microbes et plantes, il explore les propriétés nouvelles qui en émergent et modifient le fonctionnement de chaque partenaire. Il décrypte ensuite les extraordinaires adaptations symbiotiques des animaux, qu'ils soient terrestres ou sousmarins. Il décrit nos propres compagnons microbiens - le microbiote humain - et leurs contributions, omniprésentes et parfois inattendues. Enfin, il démontre le rôle des symbioses microbiennes au niveau des écosystèmes, de l'évolution de la vie et des pratiques culturelles et alimentaires qui ont forgé les civilisations.

Destiné à tous les publics, cet ouvrage constitue une mine d'informations pour les naturalistes, les enseignants, les médecins et pharmaciens, les agriculteurs, les amis des animaux et, plus généralement, tous les curieux du vivant. À l'issue de ce périple dans le monde microbien, le lecteur, émerveillé, ne pourra plus porter le même regard sur notre monde. 


\section{Sciences humaines et sociales}

Anthropologies réflexives.

Modes de connaissance et formes d'expérience

Marieke Blondet, Mickaële Lantin Mallet (Eds)

Presses universitaires de Lyon, 2017, 304 p.

L'anthropologie proposée dans cet ouvrage met au centre de sa réflexion le terrain ethnographique comme induisant, de fait, la mise en place de relations intersubjectives complexes qui participent à et pèsent sur l'enquête ainsi que sur la production du savoir. Les régimes d'engagement de l'anthropologue avant, pendant et après son enquête constituent alors un questionnement nécessaire et fondamental pour l'exercice du métier et la compréhension des mondes sociaux.

Les auteurs évoquent tour à tour comment ils se sont trouvés pris dans des interrelations qui les ont conduits à omettre certains aspects contradictoires, dérangeants ou compromettants des situations observées. Tous ont entrepris une démarche critique et réflexive sur leurs expériences de terrain et leurs rencontres et ont cherché à comprendre comment elles ont influencé à la fois leur raisonnement anthropologique et les modalités d'écriture au moment de la restitution de leur recherche.

Cette étude des situations et des relations tissées sur le terrain permet alors de comprendre comment les humains donnent sens à leur monde et décident de leurs actes. Cet ouvrage interroge ainsi, entre empirie et théorie, les modes de connaissance et les formes d'expérience au fondement du savoir anthropologique.

\section{L'emprise des marchés. Comprendre leur fonctionnement pour pouvoir les changer Michel Callon}

La Découverte, 2017, 504 p.

Alors que s'opposent avec une rare violence partisans et contempteurs de l'économie de marché, une question préalable se pose: qu'est-ce qu'un marché ?

Michel Callon montre que l'organisation des activités marchandes ne se réduit pas à la simple confrontation entre des offres et des demandes. Comment une «chose» se transforme-t-elle, après d'immenses efforts, en marchandise? Par quels moyens les agents deviennent-ils capables d'évaluer les biens et de calculer leurs décisions ? Leur rencontre n'exige-t-elle pas de méticuleux réglages? Quels sont les ressorts de la mobilisation et de l'encadrement des passions? Comment ont lieu la captation des clients et l'obtention de leur consentement à payer? À partir d'une multitude d'exemples pris dans la finance, l'énergie, l'alimentation, l'immobilier, les jeux de hasard, la santé, la grande distribution, le commerce électronique, le réchauffement climatique et même les pompes funèbres, l'auteur met en évidence la diversité et le foisonnement des activités déployées et des investissements consentis pour imaginer des solutions à ces problèmes.

$\mathrm{Au}$ terme d'un passionnant voyage, qui permet de suivre la carrière des biens depuis leur conception jusqu'à leur circulation dans la sphère de la consommation, le lecteur prend conscience de la multiplicité des configurations. Les «agencements marchands», notion que Michel Callon place au cœur même de sa réflexion et qui se substitue à celle de marché, permettent de rendre compte de la richesse et de la complexité des processus à l'œuvre. Ils donnent aussi de nouvelles possibilités d'agir à tous ceux qui refusent de se soumettre sans discussion à l'emprise des marchés existants.

\section{Le moment Gorz}

Christophe Fourel, Alain Caillé (Eds)

Le bord de l'eau, 2017, 364 p. (contient un DVD)

La pensée d'André Gorz est plus que jamais une pensée vivante. Elle fait l'objet de plus en plus de commentaires, d'analyses et d'interprétations notamment dans les milieux de l'écologie politique dont il fut un pionnier et plus largement dans tous les cercles plus ou moins militants qui cherchent les voies d'une alternative à la société actuelle dont les impasses se révèlent jour après jour plus manifestes: économie sociale et solidaire, économie circulaire, critiques de la financiarisation, convivialisme, etc.

\section{Où atterrir? Comment s'orienter en politique \\ Bruno Latour}

La Découverte, 2017, 160 p.

Cet essai voudrait relier trois phénomènes que les commentateurs ont déjà repérés mais dont ils ne voient pas toujours le lien - et par conséquent dont ils ne voient pas l'immense énergie politique qu'on pourrait tirer de leur rapprochement.

D'abord la «dérégulation » qui va donner au mot de "globalisation» un sens de plus en plus péjoratif; ensuite, l'explosion de plus en plus vertigineuse des inégalités; enfin, l'entreprise systématique pour nier l'existence de la mutation climatique.

L'hypothèse est qu'on ne comprend rien aux positions politiques depuis cinquante ans si l'on ne donne pas une place centrale à la question du climat et à sa dénégation. Tout se passe en effet comme si une partie importante des classes dirigeantes était arrivée à la conclusion qu'il n'y aurait plus assez de place sur terre pour elles et pour le reste de ses habitants. C'est ce qui expliquerait l'explosion des inégalités, l'étendue des dérégulations, la critique de la mondialisation, et, surtout, le désir panique de revenir aux anciennes protections de l'État national. 
Pour contrer une telle politique, il va falloir atterrir quelque part. D'où l'importance de savoir comment s'orienter. Et donc dessiner quelque chose comme une carte des positions imposées par ce nouveau paysage au sein duquel se redéfinissent non seulement les affects de la vie publique mais aussi ses enjeux.

\section{Science et territoires de l'ignorance}

Mathias Girel

Quæ, 2017, 160 p.

L'ignorance peut être autre chose que la pure absence de savoir ou que le simple fait d'être privé de connaissances possédées par d'autres: elle peut être surmontée, elle peut aussi être produite. Quels sont les variétés et les modes de l'ignorance, et pourquoi est-il essentiel d'en tenir compte dans les débats environnementaux et sanitaires? Lorsqu'elle est «produite», comme l'estiment certains, comment l'est-elle?

L'ouvrage répond à ces questions et, au-delà de l'opposition tranchée entre l'ignorance conçue comme front de la science et l'ignorance stratégique, il explore une véritable «zone grise» qui constitue une partie de ce paysage: conflits d'intérêts, débats sur les sources de financement de la recherche, crise de la réplication des expérimentations. Quand et comment peut-on sortir de cette «zone grise» où tout devient indiscernable pour qualifier plus nettement les phénomènes en jeu? Si nos enquêtes comme nos actions peuvent réussir ou échouer, échouer de manière épisodique ou persistante, sous l'action d'un tiers ou non, dans quels cas est-il raisonnable de relier ces échecs à des intentions? 